\title{
Electronic Structure in Narrow-Gap Quantum Dots
}

\author{
S. J. Prado ${ }^{1}$, G. E. Marques ${ }^{1}$, and C.Trallero-Giner ${ }^{2}$ \\ ${ }^{1}$ Departamento de Física, Universidade Federal de São Carlos, \\ 13565-905, São Carlos, SP, Brazil \\ ${ }^{2}$ Departamento de Física Teórica, Universidade de La Habana \\ 10400, C. Habana, Cuba.
}

Received February, 1999

\begin{abstract}
In this work we calculated the electronic structure of spherical quantum dots based on zincblend semiconductor compounds. The strong conduction-valence band coupling in this class of semiconductors induces a strong mixing of the electronic states which requires a theoretical model to properly take into acount these effects. We have used a full $8 \times 8$ Kane Hamiltonian in order to include the strong admixture and study the set of symetries associated with these electronic states and their angular momentum in this central force problem. As an application, we have calculated the electronic structure in narrow-gap $\mathrm{HgCdTe}$, InSb and CdTe quantum dots.
\end{abstract}

\section{Introduction}

The synthesis and characterization of new structural materials showing potential application as optical devices has been the subject of a large number of investigations in recent years. Almost all of them have studied semiconductor structures such as quantum wells $(\mathrm{QW}) \Gamma$ quantum well wires(QWW) and quantum $\operatorname{dots}(\mathrm{QD}) \Gamma$ presenting quantum confinement in one $\Gamma$ two and three dimensions Trespectively.

The main interest in this work will be the electronic structure of QD which show a completely discrete energy spectrum for all carriers and $\Gamma$ for this reason $\Gamma$ they are frequently referred to as "artificial atoms". Synthesis on colloids and on vitreous matrices are techniques which have shown high degree of reproducibility and control. The crystallites have uniform spherical shapes where the diameter size can be tuned during the synthesis or by thermal treatment of the vitreous matrices producing narrow distribution of QD sizes within $5 \%$ rms [1].

In these nanocrystallite structures the number of carriers in the dots can be controlled externally[2] and the full spatial confinement $\Gamma$ besides producing sharp energy levels $\Gamma$ also has strong influence on their linear
[3] and nonlinear[4] optical properties.

In this short work we show some results for the electronic structure of quantum dots with spherical symmetry within the envelope-function approximation where we solve analytically the full k.p Hamiltonian.

\section{The k.p representation}

A large number of band structure calculation has been used in solids however the k.p, first introduced by Kane[5] and Luttinger[6] has proved to be the most general effective mass approach for heterostructures where the states are expanded in a finite set of Bloch states close to an extremum $\Gamma \mathbf{k}_{0} \Gamma$ of the bulk band structure inside the Brillouin zone of the crystal.

The basic k.p Hamiltonian procedure used by us is well described by Weiler[7] and used in a modified version by [8] in QD's. We are going to use the standard k.p version Twith only a special ordering of states in two sets with spin up and down $\Gamma$ as $c^{+} \Gamma h h^{+} \Gamma l h^{+} \Gamma s o^{+} \Gamma c^{-} \Gamma$ $h h^{-} \Gamma l h^{-} \Gamma s o^{-}$. All operators appearing in the standard k.p Hamiltonian are given in terms of $k_{x}=-i \frac{\partial}{\partial x} \Gamma$ $k_{y}=-i \frac{\partial}{\partial y}$ and $k_{z}=-i \frac{\partial}{\partial z} \Gamma$ that will be transformed into spherical coordinates operators in the form

$$
\frac{\partial}{\partial x}=\sin \theta \cos \varphi \frac{\partial}{\partial r}+\cos \varphi \cos \theta \frac{1}{r} \frac{\partial}{\partial \theta}-\frac{\sin \varphi}{r \sin \theta} \frac{\partial}{\partial \varphi},
$$




$$
\begin{aligned}
\frac{\partial}{\partial y} & =\sin \theta \sin \varphi \frac{\partial}{\partial r}+\cos \theta \sin \varphi \frac{1}{r} \frac{\partial}{\partial \theta}+\frac{\cos \varphi}{r \sin \theta} \frac{\partial}{\partial \varphi} \\
\frac{\partial}{\partial z} & =\cos \theta \frac{\partial}{\partial r}-\sin \theta \frac{1}{r} \frac{\partial}{\partial \theta}
\end{aligned}
$$

Our model consider the QD with a radius $R$ and infinite barrier at the surface of the QDT since outside the crystallites the materials have amorphous structures for QD's grown on colloidal or vitreous matrices. Thus The potential is $V=0$ for $r \leq R$ and $V=\infty$ for $r>R$ is a realistic model. Therefore the solutions of

$$
\left(\widehat{H}_{k \cdot p}+V\right) \Psi_{n, l, m}(r, \theta, \phi)=E_{n, l, m} \Psi_{n, l, m}(r, \theta, \phi),
$$

can be found in the form $\Gamma$

$$
\Psi_{n, l, m}(r, \theta, \phi)=\sum_{l, n} \mathrm{C}(l, n, m) A_{n, l} J_{l}\left(\frac{\mu_{n}^{l}}{R} r\right)\left[\begin{array}{c}
Y_{l}^{m}(\theta, \phi) \mid 1> \\
Y_{l}^{m}(\theta, \phi) \mid 2> \\
Y_{l}^{m}(\theta, \phi) \mid 3> \\
Y_{l}^{m}(\theta, \phi) \mid 4> \\
Y_{l}^{m}(\theta, \phi) \mid 5> \\
Y_{l}^{m}(\theta, \phi) \mid 6> \\
Y_{l}^{m}(\theta, \phi) \mid 7> \\
Y_{l}^{m}(\theta, \phi) \mid 8>
\end{array}\right],
$$

where the term $A_{n, l} J_{l}\left(\frac{\mu_{n}^{l}}{R} r\right) Y_{l}^{m}(\theta, \phi)$ is an eigenfunction obtained as a solution $\Gamma$ for each carrier type $\Gamma$ of the diagonal elements of $\widehat{H}_{k \cdot p}$ and the kets $\{\mid j>\}$ with $(j=1,8)$ are the Bloch functions at the $\Gamma$-point $\Gamma$ with the proper symmetry of the crystallite. The coefficients $A_{n, l}$ are the normalization constants $\Gamma J_{l}\left(\frac{\mu_{n}^{l}}{R} r\right)$ are Bessel spherical functionsTas shown in Fig.1 Twhose zeroes for each level $n$ and angular momentum $l \Gamma$ are found as $\mu_{n}^{l}$ and $\Gamma$ finally $\Gamma Y_{l}^{m}(\theta, \phi)$ are the spherical harmonic functions.

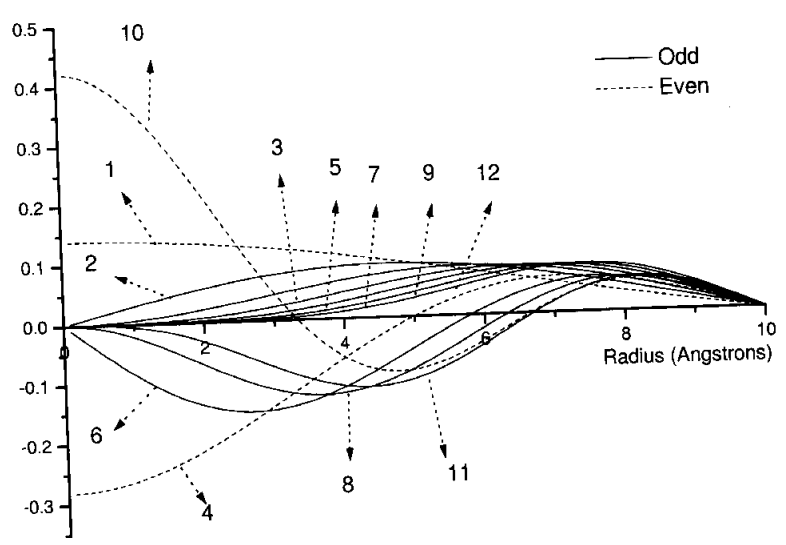

Figure 1. The first twelve spherical Bessel functions for any quantum dot of radius $R=10 \AA$. These functions have parity defined according to the angular momentum and were chosen in order of increasing energy.
The numberTin the Fig. $1 \Gamma$ labels the first twelve spherical Bessel functions in order of increasing energy. The are associated to each root of $J_{l}\left(\frac{\mu_{n}^{l}}{R} r\right) \Gamma$ which has quantum numbers $(n \Gamma l)$ for each energy level having degeneracy $(m=2 l+1)$ in the quantum number $m$. The root are only function of $(n, l)$ and $\Gamma$ in a spectroscopic notation they represent the $(n l)$-atomic like states $\Gamma$ and are labeled: $1 S: l=0 ; 1 P: l=1 ; 1 D: l=2$; $2 S: l=0 ; 1 F: l=3 ; 2 P: l=1 ; 1 G: l=4 ; 2 D: l=2$; $1 H: l=5 ; 3 S: l=0 ; 2 F: l=3 ; 1 I: l=6$.

Within the model used in this work every element of matrix $\Gamma<\psi_{n^{\prime}, l^{\prime}, m^{\prime}}\left|\widehat{H}_{k, p}\right| \psi_{n, l, m}>$ Tin the spherical QD'sTcan be calculated analytically since every part in $\psi_{n, l, m}(r, \theta, \phi)$ is a well known function.

\section{Results}

We will apply our model to calculate the QD electronic structure $\Gamma$ as a function of the radius $\Gamma$ in three different materials. We have chosen to make the comparison of the energy levels of QD's for CdTeT InSbT $H g_{0.8} \mathrm{Cd}_{0.2} \mathrm{Te}$ due to the size of their band gap energy. The Table below show all values of all $\mathbf{k}$.p parameters used in the calculation. 


\begin{tabular}{|l|l|l|l|}
\hline Parameters & $H g C d T e^{(b)}$ & $\operatorname{InSb}^{(a)}$ & $C d T e^{(a)}$ \\
\hline$F$ & -0.80 & -0.68 & -0.50 \\
\hline$E_{g} \quad(m e V)$ & 75.98 & 236.8 & 1606.9 \\
\hline$\Delta_{s o} \quad(m e V)$ & 1000.0 & 810.0 & 920 \\
\hline$\gamma_{1}$ & 3.30 & 3.440 & 1.657 \\
\hline$\gamma_{2}$ & 0.10 & -0.524 & -0.817 \\
\hline$\gamma_{3} \quad(m e V \times \bar{A})$ & 85.083 & 94.462 & 82.600 \\
\hline$P$ & $(b) \operatorname{Ref} .[7]$ & $\bar{A}=100 A$ & \\
\hline$(a)$ Ref.[9] & \multicolumn{3}{|l}{} \\
\hline
\end{tabular}

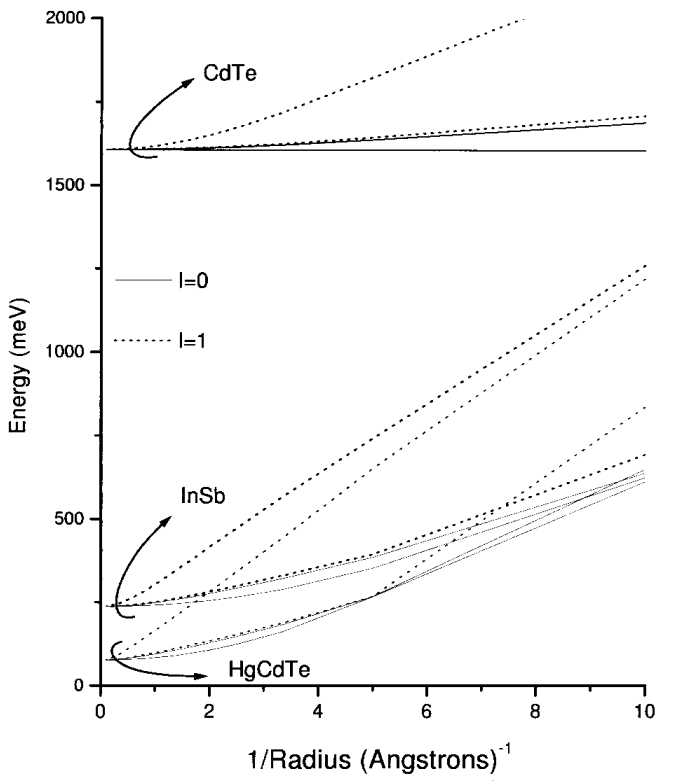

Figure 2. Comparison of the effect due to conductionvalence band coupling on the first four energy levels of electrons in quantum dots of the three materials listed in Table I.

In Fig. 2 we show the conduction band states $\Gamma$ for each material of Table ITas a function of the inverse of the radius of the QD. We display the first four states $\Gamma$ being two for $l=0$ and two for $l=1$. It becomes very clear that the coupling between the conduction and the valence bands is the strongest one for $\mathrm{HgCdTe}$ material $\Gamma$ as should be expected due to its very small band gap energy. This effect is somewhat similar for $\operatorname{In} S b$ and becomes smallibut not negligible at allTfor $C d T e$ QD's.

In Fig. 3 we show the calculated $\Gamma_{8}$ valence band energy spectra for $l=0$ and $l=1 \Gamma$ as a function of the inverse of the radius of the QDTfor all three materials of Table I. Here again the two material with narrow gap somewhat show similar dispersions for the mixed $l h$ and $h h$ states. For $C d T e$ the energy levels corresponding to $l=0$ are further away from those with $l=1$.

Finally we may notice the complexity of the valence band electronic structure in spherical quantum dots. It seems that our full k.p Hamiltonian model can describe well these states and some degeneracy are lifted for some quantum numbers. We intend to further explorer and study these effects on the optical spectrum of these structures.

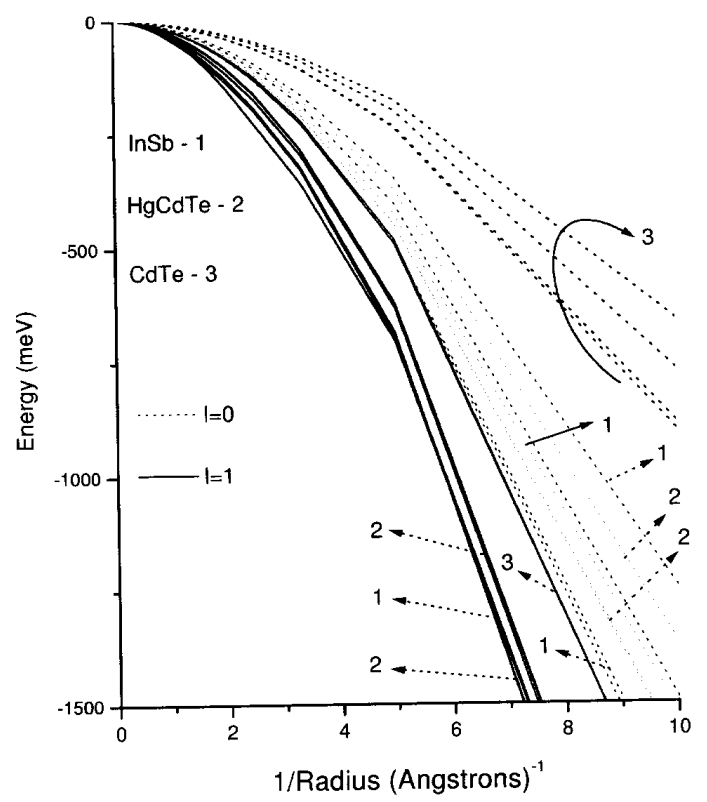

Figure 3. Comparison of the effect due to valence band mixing on the first four energy levels of light- and heavy-holes in quantum dots of the three materials listed in Table I.

Authors acknowledges Conselho Nacional de Desenvolvimento Científico e Tecnológico( $\mathrm{CNPq})$ and Fundação de Amparo à Pesquisa do Estado de São Paulo(FAPESP) for partial financial support of this work.

\section{References}

[1] C. B. Murray, D. J. Norris, and M. G. Bawendi, J. Am. Chem. Soc. 115, 8706 (1993).

[2] J. H. Oh, K. J. Chang, G. Ilm, S. J. Lee, Phys. Rev.B 53, 13264 (1996) 
[3] Tristan Richard, Pierre Lefebvre, Henry Mathieu, Jacques Allégre, Phys. Rev.B 53, 7287 (1996).

[4] S. Schmitt-Rink, D. A. B. Miller, D. S. Chemla, Phys. Rev.B 35, 8113 (1987).

[5] E. O. Kane, Semiconductor and Semimetals, Willardson, R.K. e Beer, A.C., eds. (Academic Press, New York, 1966), vol. 1, p.75.

[6] J. M. Luttinger, W. Kohn, Phys. Rev. 97, 869 (1955).

[7] M. H. Weiler, Semiconductor and Semimetals, Willardson, R.K. e Beer, A.C., eds. (Academic Press, New
York, 1981), vol. 16, p.119.

[8] Peter C. Sercel, Kerry J. Vahala, Phys. Rev. B42, 3690 (1990).

[9] Semiconductors: Physics of II-VI and I-VII Compounds, Semimagnetic Semiconductors, Vol. 17(b) of Landolt-Börnstein, Numerical Data and Functional Relationships in Science and Technology, New Series, edited by O. Madelung, M. Schulz, and H. Weiss (Springer-Verlag, Berlin Heidelberg, 1982). 